\title{
MR to Ultrasound Image Registration for Guiding Prostate Biopsy and Interventions
}

\author{
Yipeng $\mathrm{Hu}^{1}$, Hashim Uddin Ahmed ${ }^{2}$, Clare Allen ${ }^{3}$, Doug Pendsé ${ }^{3}$, Mahua Sahu ${ }^{2}$, \\ Mark Emberton ${ }^{2}$, David Hawkes ${ }^{1}$, and Dean Barratt ${ }^{1}$ \\ ${ }^{1}$ Centre for Medical Image Computing, University College London, London, UK \\ ${ }^{2}$ Department of Urology, Division of Surgery \& Interventional Science, University College \\ London, London, UK \\ ${ }^{3}$ Department of Radiology, University College London Hospital (UCLH), London, UK
}

\begin{abstract}
A method is described for registering preoperative magnetic resonance (MR) to intraoperative transrectal ultrasound (TRUS) images of the prostate gland. A statistical motion model (SMM) of the prostate is first built using training data provided by biomechanical simulations of the motion of a patientspecific finite element model, derived from a preoperative MR image. The SMM is then registered to a 3D TRUS image by maximising the likelihood of the shape of an SMM instance given a voxel-intensity-based feature, which represents an estimate of normal vector at the surface of the prostate gland. Using data acquired from 7 patients, the accuracy of registering T2 MR to 3D TRUS images was evaluated using anatomical landmarks inside the gland. The results show that the proposed registration method has a root-mean-square target registration error of $2.66 \mathrm{~mm}$.
\end{abstract}

\section{Introduction}

Prostate cancer is a major international health problem, particularly affecting men in the Western World. Traditional treatment strategies for localised prostate cancer involve either radical treatment of the whole gland - for example, by surgical excision or radiotherapy - or pursuing an active surveillance/watchful waiting programme in which intervention is delayed in favour of monitoring the patient for signs of disease progression. Motivated by the potential for a reduced risk of side-effects compared with conventional treatments, there has recently been growing interest in techniques which enable the targeted treatment of prostate cancer and minimise damage to vulnerable structures, such as nerves [1]. However, accurate mapping of cancer foci and accurate therapy delivery are of critical importance in such approaches.

Transrectal ultrasound (TRUS) imaging remains the most accessible and practical means for guiding needle biopsy and therapeutic interventions. However, has poor accuracy for visualisation of cancer foci within the prostate means conventional (Bmode) TRUS imaging provides very limited information on the spatial location of tumours. Consequently, TRUS images are not suitable for disease mapping, and performing accurate, targeted biopsy. Instead, current standard of care is to use TRUS to simply inform the operator that the needle is in the prostate and which side it is in. Recent advances in functional and structural MR imaging techniques for localising 
and characterising prostate cancer have led to sensitivities and specificities that are now sufficiently high to be clinically useful for guiding biopsy needles and for targeting localised therapy in a more precise manner [2]. However, the ability to fuse anatomical and pathological information on tumour location, derived from MR images or a previous biopsy, with TRUS images obtained during a procedure remains a significant technical challenge.

In this paper, a new method is presented for non-rigid registration of MR images and 3D TRUS images, which compensates for gland motion and is sufficiently fast for intraoperative use. The method uses a previously demonstrated approach that combines finite element analysis (FEA) and statistical shape modelling to generate a compact model of prostate gland motion due to insertion of a TRUS probe into the rectum $[3,4]$. In this study, this technique is used to construct patient-specific, biomechanically-informed statistical motion models (SMMs) from preoperative MR images in order to predict physically realistic deformations as well as providing a wellconstrained transformation model for non-rigid registration of MR and TRUS images.

Related previous work reported by $\mathrm{Wu}$ et al. [5] and $\mathrm{Xu}$ et al. [6] was limited to rigid registration, and is therefore unable to compensate for the significant gland deformation that commonly occurs between MR and TRUS imaging. In the latter study, an initial manual MR-TRUS registration is also required, and quantitative accuracy results are only presented for a phantom [6]. In this work, we propose a "model-toimage" registration approach in which a deformable model of the prostate gland, constructed from segmented MR images prior to a procedure, enables rapid registration to TRUS images acquired during the procedure with minimal user interaction. Although there is a significant time overhead associated with generating such a model, much of the preoperative processing is performed automatically and the level of user interaction required is feasible for the purposes of surgical planning, especially if advanced image segmentation tools are employed.

\section{Methods}

An overview of the registration method developed in this work is illustrated in Fig. 1. The following two-stage scheme is proposed:

a) Planning Stage (before a surgical procedure): i) Build a patient-specific finite element $(\mathrm{FE})$ mesh of the prostate gland and surrounding anatomy from preoperative

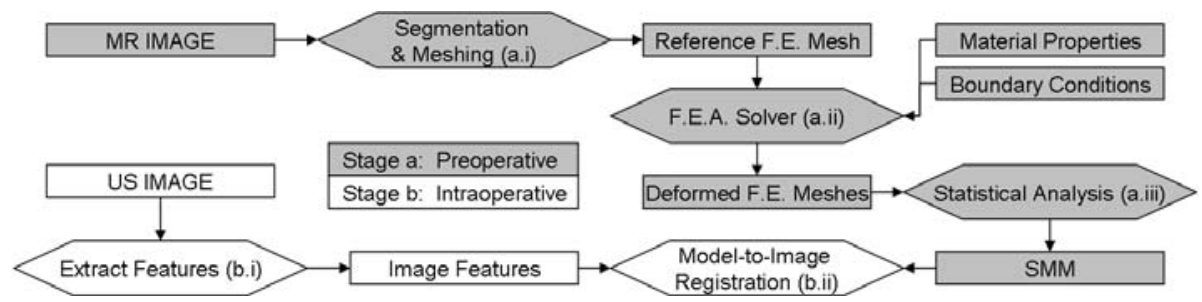

Fig. 1. An overview of the registration method. Preoperative processes and data are shaded grey, whereas intraoperative processes and data are not shaded. 
MR image; ii) Perform a series of FEA simulations of gland motion using randomly sampled material properties and boundary conditions; iii) Construct a SMM by applying principal component analysis (PCA) to the predicted FE mesh node displacements.

b) Registration Stage (during a procedure): i) Compute the TRUS image feature vectors by computing second derivatives of the image intensity; ii) iteratively optimise the rigid-body and SMM shape parameters until the likelihood of a particular set of registration parameters given the TRUS image is maximised.

In Section 2, a detailed description of these two stages is provided.

\subsection{Statistical Motion Model Generation}

For the purposes of this study, diagnostic MR images were manually segmented into regions, which defined the geometry of the prostate gland (divided anatomically into the central and peripheral zones), the pelvis bone, the rectum and the bladder at the base of the prostate (see Fig. 2). The prostate gland was initially described using a spherical harmonic representation, which was then converted into a triangulated surface mesh. The lower part of the pelvis was also meshed. The surfaces were then imported into the commercial FEA software package ANSYS (ANSYS Europe Ltd., Oxfordshire, UK) and a FE model constructed with 50-60,000 tetrahedral elements using the solid modeling tools provided by the software. Ten-node tetrahedral elements were used as these support non-linear geometries using unstructured meshes. The mesh was refined around the region of rectum so that the TRUS probe could be modeled directly in simulations without remeshing.

Elements within all regions of interest were labeled and each assigned material properties randomly sampled from a physiological range. All tissues were assumed to behave as isotropic, linear elastic materials. Since it can been argued that the assumption of incompressibility (Poisson's ratio, $v=0.5$ ) may not be appropriate for organs such as the prostate because of gain and loss of blood and other fluids, and the presence of a collapsible urethra, both the Young's modulus and the Poisson's ratio assigned to different materials in the FE model were assumed to be unknown and therefore sampled randomly in the simulations.

Boundary conditions for each FEA simulation were determined as follows: The displacement on the surface of the pelvis was set to zero for all simulations. A random configuration of the TRUS probe in terms of its pose and the diameter of the waterfilled sheath were set for each simulation [4].

After assigning sampled material properties and boundary conditions for each of 500 simulations, the node displacements were computed using the preconditioned conjugate gradient iterative equation solver in ANSYS.

Since correspondence was established between the deformed prostate models, PCA was applied directly to the 3D displacements of the mesh nodes, as follows: For each of $M$ (= 500) simulated gland deformations, the displacement of each of $N$ nodes in the prostate gland mesh was calculated and combined to form a $3 N \times 1$ vector d, which describes the predicted motion of the prostate gland for a particular set of material properties and boundary conditions. The principal modes of variation in $\mathbf{d}$ were then calculated using PCA. If $\mathbf{m}_{0}$ represents the undeformed gland and is a vector containing the 3D coordinates of the nodes of the original FE model determined from the MR image, then a deformed gland is defined by vector $\mathbf{m}$, given by: 

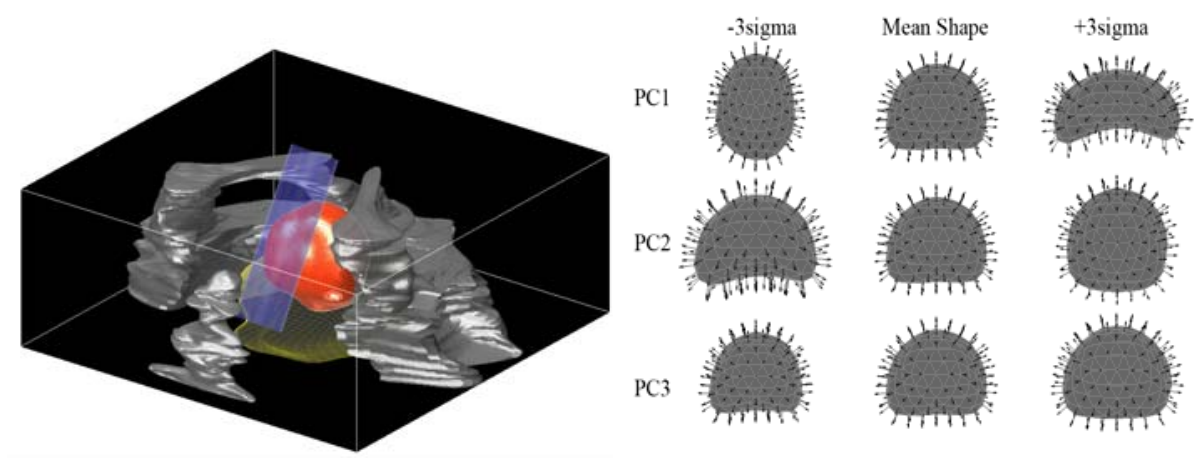

Fig. 2. Left: An illustration of surface meshes derived from an MR image. The TRUS probe (with sheath) is approximated by a cylinder (shown in blue). Right: The first 3 modes (PC1, $\mathrm{PC} 2 \& \mathrm{PC} 3)$ of an SMM showing the variation in prostate shape. The normal vectors at the nodes of the triangulated surfaces are indicated by arrows.

$$
\mathbf{m}=\mathbf{m}_{0}+\overline{\mathbf{d}}+\sum_{i=1}^{L} c_{i} \mathbf{e}_{i}
$$

where $\overline{\mathbf{d}}$ is the mean node displacement vector, $\mathbf{e}_{\mathbf{i}}$ is the $i^{\text {th }}$ eigenvector of the covariance matrix, and $c_{i}$ is a scalar weight. $L \leq M$ was chosen so that the SMM covered $>99 \%$ of variance in the training data, typically, $L \sim 15$. Additionally, the normal vectors at the nodes (vertices) of the triangulated surface were computed. An example of the shape changes of a prostate model corresponding to the first three modes of the shape variation, are illustrated in Fig. 2.

\subsection{Ultrasound Surface Feature Detection}

One distinct feature in MR and TRUS images of the prostate gland is the capsule surface. In the proposed registration method, vector representations of this surface, computed independently from the MR-derived model and the 3D TRUS image, are used to drive the model-to-image registration by maximising the similarity between these vectors. In this formulation, the surface of a deformable model, given a set of registration parameters (i.e. rigid-body parameters and shape parameters defined by $\left\{c_{1}\right.$, $\left.\left.c_{2}, \ldots, c_{L}\right\}\right)$, is uniquely defined by the surface normal vector field $\mathbf{u}(\mathbf{x})$, where $\mathbf{x}$ is a position vector that defines the $3 \mathrm{D}$ co-ordinates of a point in the model space, and $\mathbf{u}$ is a $3 \mathrm{D}$ vector function that defines the surface normal at a particular point. By definition $\mathbf{u}$ is zero at all points not lying on the model surface.

Similarly, a surface normal vector field, denoted by $\mathbf{v}$, can be estimated for the image using a multiscale filtering technique based on second-order Gaussian derivatives. In such approaches, the Hessian is computed at each voxel for a particular scale. The relative magnitudes of the eigenvalues of the Hessian can then be used to classify the local structure, enhancing blob-, tubular- or sheet-like structures [7]. In this work, an extension of the sheet-like enhancement filter proposed by Descoteaux et al. [7] was derived to take into account the non-uniform ultrasound (US) image intensity characteristics found at boundaries due to the variable angle between a boundary surface and the US beam path. This effect is responsible for artefacts where, for example, the 

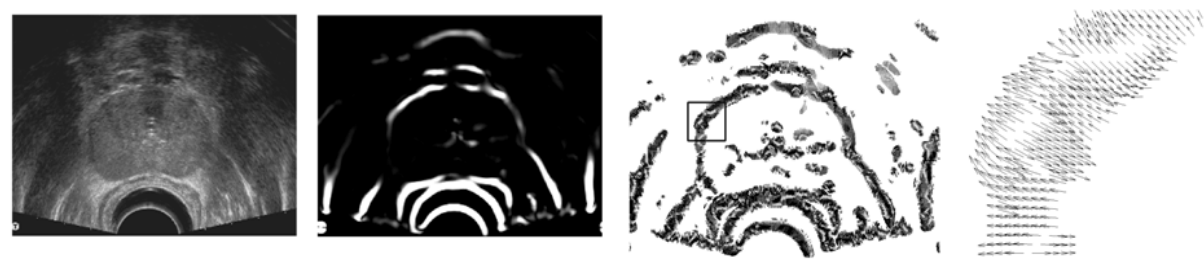

Fig. 3. Example of the surface normal vector field estimated from a 3D TRUS image using the method described in Section 2.2. From left to right: transverse slice through the original TRUS volume; image representing the response of the filter defined in Eq. (3); extracted vector field $\mathbf{v}$ (projected onto the slice) given by Eq. (4); and a zoomed-in view of a region of interest (shown in the third image) around part of the gland surface.

intensities at the boundary on the lateral sides of the prostate gland are low compared to those on the inferior and superior sides of the gland.

In the original formulation described in [9], the filter response is given by:

$$
f_{\text {sheet }}(x, y, z)=\exp \left(-\frac{\left(R_{1}\right)^{2}}{2 \alpha^{2}}\right)\left(1-\exp \left(-\frac{\left(R_{2}\right)^{2}}{2 \beta^{2}}\right)\right)\left(1-\exp \left(-\frac{\left(R_{3}\right)^{2}}{2 \gamma^{2}}\right)\right),
$$

where, the ordered eigenvalues $\lambda_{1}, \lambda_{2}$ and $\lambda_{3}\left(\left|\lambda_{1}\right| \leq\left|\lambda_{2}\right| \leq\left|\lambda_{3}\right|\right)$, of the Hessian, were computed at point $(x, y, z), R_{1}=\left|\lambda_{2}\right| \lambda_{3}\left|, R_{2}=\right| 2\left|\lambda_{3}\right|-\left|\lambda_{2}\right|-\left|\lambda_{1}\right| \mid$ and $R_{3}=\left(\lambda_{1}{ }^{2}+\lambda_{2}{ }^{2}+\lambda_{3}{ }^{2}\right)^{0.5}$. For the TRUS data collected in this study, the response of this filter was found to be insensitive to the scalar weighting parameters $\alpha, \beta$ and $\gamma$, and therefore these were set to constant values as suggested in [7]. The width $\sigma$ of the Gaussian kernel used to compute the Hessian was $1 \mathrm{~mm}$ in all directions.

If the direction of the US beam is defined by the 3D vector $\mathbf{b}$, then the modified filter response is given by:

$$
f_{\text {sheet }}^{*}=\left(\mathbf{n}_{3}^{T} \mathbf{b}\right)^{2} f_{\text {sheet }},
$$

where $\mathbf{n}_{3}(x, y, z)$ is the normalised eigenvector corresponding to the largest eigenvalue $\left(\lambda_{3}\right)$ of the Hessian, which will be approximately co-linear with the surface normal at the surface. The weighting factor in Eq. (3) reduces the response to noise when direction of the US beam is approximately perpendicular to the surface normal. Finally, the surface normal vector field is given by:

$$
\mathbf{v}(x, y, z)=\left\{\begin{array}{cc}
\mathbf{n}_{3}, & \text { if } a \leq f_{\text {sheet }}^{*}(x, y, z) \leq b \text { and } \lambda_{3}>0 \\
\mathbf{0}, & \text { otherwise }
\end{array}\right.
$$

where the scalars $a$ and $b$ specify a window in which the filter response is considered to be significant. An example of this vector field is given in Fig.3.

\subsection{Definition of a Probability Density Function for the Surface Noise Model}

To enable robust model-to-image registration, a similar approach to that described by Staib and Duncan [8] was adopted. The model-to-image registration problem is equivalent to the boundary finding problem considered in [8], and a feature extracted from the image, such as the surface normal vector field, described above, may be considered to be a noise-corrupted version of the surface normal vector field determined from the deformable model $\mathbf{m}$ (defined in Section 2.1). In this formulation, the probability that a particular image voxel, referenced by the index $i$ in the image space 
$\Omega_{\text {image }}$, has co-ordinates $\mathbf{y}_{i}=\left(x_{i}, y_{i}, z_{i}\right)$ and an estimated surface normal vector $\mathbf{v}_{i}$ can be expressed as a probability mixture model as follows:

$$
f_{N}\left(\mathbf{y}_{i}, \mathbf{v}_{i} \mid \mathbf{m}\right)=\sum_{j \in \Omega_{\text {model }}} h_{j} f_{\mathrm{G}}\left(\mathbf{y}_{i} ; \mathbf{x}_{j}\right) f_{\mathrm{W}}\left(\mathbf{v}_{i} ; \mathbf{u}_{j}\right)
$$

where $h_{j}$ is a mixing parameter, $\Sigma h_{j}=1, j$ is an index to a discrete point in the model space $\Omega_{\text {model }}$, defined by $\mathbf{x}_{j}=\left(x_{j}, y_{j}, z_{j}\right)$, and $f_{G}$ and $f_{W}$ are probability density functions that describe Gaussian and bipolar Watson distributions [9] defined as:

$$
f_{G}\left(\mathbf{y}_{i} ; \mathbf{x}_{j}\right)=\left((2 \pi)^{1.5}\left|\boldsymbol{\Sigma}_{j}\right|^{0.5}\right)^{-1} \exp \left(-\frac{1}{2}\left(\mathbf{x}_{j}-\mathbf{y}_{i}\right)^{\mathbf{T}} \boldsymbol{\Sigma}_{j}^{-\mathbf{1}}\left(\mathbf{x}_{j}-\mathbf{y}_{i}\right)\right)
$$

and

$$
f_{W}\left(\mathbf{v}_{i} ; \mathbf{u}_{j}\right)=C(k) \exp \left(k\left(\mathbf{u}_{j}^{\mathbf{T}} \mathbf{v}_{i}\right)^{2}\right)=C(k) \exp \left(k \cos ^{2} \theta\right),
$$

respectively.

In Eq. (6) a special class of anisotropic Gaussian with two parameters is used where the covariance matrix $\Sigma_{j}$ is restricted by a set of orthogonal vectors $\mathbf{w}_{d}$ :

$$
\boldsymbol{\Sigma}_{j}=\sum_{d=1}^{3} \rho_{d} \mathbf{w}_{d} \mathbf{w}_{d}{ }^{\mathbf{T}},
$$

where $\mathbf{w}_{d}$ defines the orientations of the ellipsoid (which defines a surface of constant probability density), and $\mathbf{w}_{1}$ is set to $\mathbf{u}_{j}$. The two independent parameters, $\rho_{1}$ and $\rho_{2}$ $\left(=\rho_{3}\right)$ govern the "capture range" in the surface normal direction and in the tangent plane, respectively. For the experiments described here, $\rho_{1}=2 \rho_{2}$.

In Eq. (7) $k$ is a scalar concentration parameter, which is varied depending on the noise level. $k$ was set to a small value $0.1<k<0.5$ in order to weaken the contribution from strong local match. The normalising constant $C(k)$ was estimated by recursive integration to satisfy the requirements of a probability density function. $\theta$ is the angle between the model surface normal vector, computed at point $j$, and the image surface normal vector, computed at voxel $i$.

\subsection{Registration Scheme}

The aim of the registration algorithm is to find the optimal registration parameters which maximise the joint probability of the noise. Assuming that the noise values at different voxels are independent [8], we arrive at the following log likelihood objective function:

$$
\begin{aligned}
f_{o b j} & =\log (L(\mathbf{m} \mid \mathbf{I}))=\log \prod_{\Omega_{\text {image }}} P(\mathbf{I} \mid \mathbf{m})=\log \prod_{i \in \Omega_{\text {image }}} f_{N}\left(\mathbf{y}_{i}, \mathbf{v}_{i} \mid \mathbf{m}\right) \\
& =\sum_{i \in \Omega_{\text {image }}} \log \sum_{j \in \Omega_{\text {model }}} h_{j} f_{G}\left(\mathbf{y}_{i} ; \mathbf{x}_{j}\right) f_{W}\left(\mathbf{v}_{i} ; \mathbf{u}_{j}\right)
\end{aligned}
$$

The expectation maximisation (EM) algorithm provides an efficient means of maximising a likelihood function [10]. An EM algorithm was implemented using Matlab (The Mathworks Inc.), which iteratively optimises the registration parameters in order to maximise Eq. (9).

\section{Experiments and Validation Results}

All patient data used in this study was obtained from patients recruited to clinical research studies at University College London Hospital (UCLH), approved by the local 
Table 1. Target registration errors (TREs) calculated for intra-prostatic landmarks

\begin{tabular}{cccccccccc}
\hline \multicolumn{2}{c}{ Case No. } & $\mathbf{1}$ & $\mathbf{2}$ & $\mathbf{3}$ & $\mathbf{4}$ & $\mathbf{5}$ & $\mathbf{6}$ & $\mathbf{7}$ & All \\
\hline Number of Landmarks & 5 & 3 & 3 & 4 & 4 & 4 & 3 & 26 \\
\hline TRE (mm) & RMS & 1.92 & 3.67 & 3.14 & 1.86 & 1.57 & 3.23 & 3.12 & 2.66 \\
& Mean & 1.84 & 3.01 & 3.02 & 1.07 & 1.47 & 3.21 & 2.91 & 2.36 \\
& Std. & 0.63 & 2.58 & 1.04 & 0.94 & 0.64 & 0.46 & 1.38 & 1.24 \\
& Max. & 2.53 & 5.98 & 4.19 & 2.93 & 2.36 & 3.56 & 4.46 & 5.98 \\
\hline
\end{tabular}

research ethics committee. All patients gave written consent to participate. Data from 7 patients with prostate cancer were used to validate the method described here. T2weighted MR image volumes of the prostate gland were acquired prior to templateguided transperineal needle biopsy under general anaesthesia. Immediately before needle insertion, 3D TRUS images of the gland were acquired using a B-K ProFocus scanner (B-K Medical Ltd., Berkshire, UK). A set of parallel 2D transverse B-mode images were captured at $2 \mathrm{~mm}$ intervals and stored on the scanner. A mechanical stepping device (Tayman Medical Inc., MO, USA) was used to translate the US probe (B-K 8658T, 5-7.5 MHz transducer) perpendicular to the axial plane along the rectum.

Each US image was first resampled into a volume with an isotropic voxel dimension of $1 \mathrm{~mm}$. At each voxel, the Hessian was computed in the frequency domain using an implementation based on the fast Fourier transform. A quick and simple procedure was used to initialise the pose of SMM with respect to the TRUS volume where two points at the apex and base of the gland were manually identified. Once registered, a dense displacement field (DDF) was computed across the volume of interest by interpolating the final instance of the SMM with a solid FE mesh using a shape function for tetrahedral elements.

Corresponding anatomical landmarks, including cysts, calcifications, the urethra, the puboprostatic ligament, and the junction between the seminal vesicles, the vas deferens and the midline of the gland, were identified manually in both the MR and TRUS volumes. The 3D co-ordinates of landmarks defined in the MR image were then propagated into TRUS co-ordinates using the DDF. For each pair of identified and propagated landmarks, a target registration error (TRE) was calculated, defined as the distance between the manually defined and propagated landmarks in the TRUS space. The MR images were also warped using the DDF to allow a visual assessment of the registration.

The landmark-based results are given in Table 1. The root-mean-square (RMS) TRE over all 7 cases (26 landmarks) was $2.66 \mathrm{~mm}$. Fig. 4 illustrates the warped MR images and target TRUS images computed from an example registration.

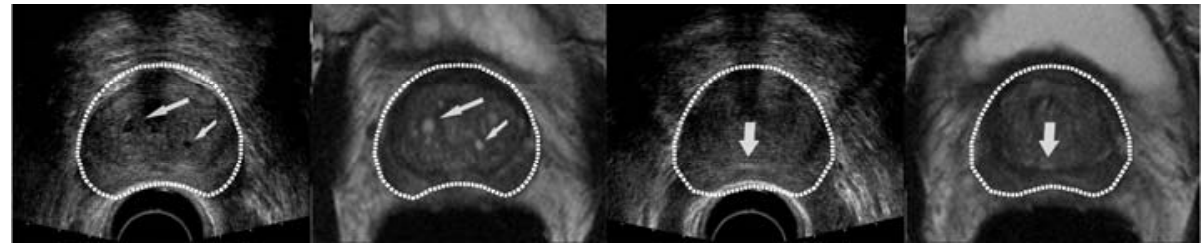

Fig. 4. Example transverse image slices $\left(1^{\text {st }}\right.$ and $3^{\text {rd }}$ images) through a TRUS volume for Case 1 shown with the corresponding warped MR images $\left(2^{\text {nd }}\right.$ and $4^{\text {th }}$ images) following deformable registration. The arrows indicate landmarks which were well aligned. 


\section{Discussion}

A model-to-image registration method is proposed in this study, which uses a combined statistical-biomechanical model built from an MR image. Building the model requires manual segmentation of the MR image and is computationally intensive, but is performed preoperatively, so does not significantly impact the intraoperative workflow. A model to image registration can currently be performed within 2 minutes using a desktop PC with a $2.33 \mathrm{GHz}$ Intel ${ }^{\circledR}$ Core $^{\mathrm{TM}}$ dual $\mathrm{CPU}$ processor and $3 \mathrm{~GB}$ of RAM. The method achieved sufficiently high accuracy to be clinically useful for MR-targeted prostate biopsy and interventions. Although only the gland surface is registered in this scheme, the use of a deformable finite-element model enables the displacement of internal structures to be rapidly computed.

Acknowledgments. This work is funded by Royal Academy of Engineering/EPSRC Research Fellowship and UCLH/UCL Comprehensive Biomedical Research Centre. The authors would like to thank Tim Carter, Gerard Ridgeway and Xiahai Zhuang for helpful ideas and discussions.

\section{References}

1. Ahmed, H.U., Emberton, M.: Active Surveillance and Radical Therapy in Prostate Cancer: Can Focal Therapy Offer the Middle Way? World J. Urol. 26, 457-467 (2008)

2. Kirkham, A.P.S., Emberton, M., Allen, C.: How good is MRI at detecting and characterising cancer within the prostate? Eur. Urol. 50, 1163-1174 (2006)

3. Mohamed, A., Davatzikos, C., Taylor, R.: A combined statistical and biomechanical model for estimation of intra-operative prostate deformation. In: Dohi, T., Kikinis, R. (eds.) MICCAI 2002. LNCS, vol. 2489, pp. 452-460. Springer, Heidelberg (2002)

4. Hu, Y., Morgan, D., Ahmed, H.U., Pendsé, D., Sahu, M., Allen, C., Emberton, M., Hawkes, D., Barratt, D.: Modelling Prostate Gland Motion for Image-guided Interventions. In: Bello, F., Edwards, E. (eds.) ISBMS 2008. LNCS, vol. 5104, pp. 79-88. Springer, Heidelberg (2008)

5. Wu, R., Ling, K.V., Shao, W., Ng, W.-S.: Registration of Organ Surface with Intraoperative 3D Ultrasound Image Using Genetic Algorithm. In: Ellis, R.E., Peters, T.M. (eds.) MICCAI 2003. LNCS, vol. 2878, pp. 383-390. Springer, Heidelberg (2003)

6. Xu, S., Kruecker, J., Turkbey, B., Glossop, N., Singh, A.K., Choyke, P., Pinto, P., Wood, B.J.: Real-time MRI-TRUS fusion for guidance of targeted prostate biopsies. Comput. Aided Surg. 13(5), 255-264 (2008)

7. Descoteaux, M., Audette, M.A., Chinzei, K., Siddiqi, K.: Bone Enhancement Filtering: Application to Sinus Bone Segmentation and Simulation of Pituitary Surgery. In: Duncan, J.S., Gerig, G. (eds.) MICCAI 2005. LNCS, vol. 3749, pp. 247-255. Springer, Heidelberg (2005)

8. Staib, L.H., Duncan, J.S.: Boundary Finding with Parametrically Deformable Models. IEEE Trans. PAMI 14, 1061-1075 (1992)

9. Watson, G.S.: Statistics on Spheres. Wiley, New York (1983)

10. Figueiredo, A., Gomes, P.: Performance of the EM Algorithm on the Identification of a Mixture of Watson Distributions Defined on the Hypersphere. Statistical Journal 4(2), $111-130$ (2006) 